\title{
Composition of Trees Grown Surrounding Water Springs at Two Areas in Purwosari Pasuruan, East Java
}

\author{
Soejono
}

Purwodadi Botanic Garden-Indonesian Institute of Sciences

\begin{abstract}
The aim of the research was to find out the composition of trees grown surrounding water springs at two areas in Purwosari, Pasuruan, East Java. Eleven plots for each area were observed. The data were analyzed using Mueller-Dombois's method to calculate their importance value indexes, while Shannon-Wiener's formula was used for determining the diversity index. The coordinate and altitude of every water spring or its group's site was determined using Geographical Position System (GPS) to know their positions on the map. The result indicated that there were at least 30 families, 49 genera which consisted of 68 species of trees grown surrounding water springs at the first area with 5.49 of diversity index, while the second area, consisted of 34 families, 63 genera and 79 species of trees with 5.24 diversity index. The diversity of trees species from Moraceae was the highest among other families, both at the first and the second area, whereas, trees species having a significant important value index included Bambusa blumeana, Dendrocalamus asper, Ficus racemosa, Horsfieldia irya and Ficus virens. The position of the springs in the two areas within the sub-districts of Purwosari is in the range of $7^{\circ} 44^{\prime} 448$ " south latitude; $112^{\circ} 44^{\prime} 353^{\prime \prime}$ east longitude up to $7^{\circ} 46^{\prime} 339$ " south latitude; $112^{\circ} 41^{\prime} 190^{\prime \prime}$ east longitude at an altitude between 251 and $522 \mathrm{~m}$ above sea level. We hope that the information can be used as an alternative guidance to restore or to conserve such areas in order to save and sustain trees diversity and their habitat.
\end{abstract}

Keywords: composition, trees, water springs, Purwosari, Pasuruan.

\section{BACKGROUND}

Plants play a fundamental role in maintaining the basic ecosystem functions and the quality of life on earth. Not only do they produce bredthable oxygen, but they also take up carbon dioxide as they photosynthesize. All life depends on the ability of plants to capture the energy in sunlight and to convert it into the chemical energy. They provide the essential things for humans well-being: the varied food, the medicines, the materials with which we build our homes, fashion of our clothing, and the fuel that powers our energy. Plants are also an integral part of the processes that distribute and purify the water. They help to hold soil in place and to renew its fertility, provide natural food control, detoxify and decompose waste, and to purify the water and stabilize the climate.

In general, different plant species have differ-

*Corresponding address:

Soejono

Purwodadi Botanic Garden-Indonesian Institute of Sciences,

Pasuruan, Indonesia

Email: soejono@lipi.go.id rent requirements in term of climate, soil type aspects and their essential biological relationships. For these reasons, the different plants communities are found in different habitats [1]. However, some plant species have a wide tolerance ranging their requirements; often, we find communities that vary in the same habitat, or the vice versa. Viewed from the conservation aspect, because of habitat destruction that has been reported elsewhere, a study on trees composition grown surrounding water springs is necessary. Based on the results of trees composition study, various follow-up activities can be done, for example the selection species, phenological studies, propagation and development of seedlings for reforestation or restoration. Thus, the expected quality of greening can be increased based on the advance and actual data, so that the multifunctional role of greening not only serves as a conservation area but also serves as a conservation of local species diversity, conservation of springs, improvement in the uptake of carbon dioxide and sustainable habitat restoration. The aim of the research was to find out the composition of 
trees grown surrounding water springs at two areas in Purwosari, Pasuruan, East Java.

\section{MATERIALS AND METHODS}

The two areas observed are administratively located in Purwosari, Pasuruan. The first area is located in the village of Sekarmojo-Cendono and the second is located in the village of PucangsariPager-Kucur. Eleven plots for each area were observed. The data were analyzed using MuellerDombois's method to calculate the importance value index, while Shannon-Wiener's formula was used for determining the diversity index [2]. The coordinate and altitude of every water springs or its group's site was determined using Geographical Position System (GPS) to know the elevation of water spring places and their positions on the map.

\section{RESULT AND DISCUSSION}

The results of the study indicated that at least 30 families, 49 genera which consisted of 68 species of trees grown surrounding water springs at the first area with 5.49 of diversity index, while the second area consisted of 34 families, 63 genera and 79 species of trees with 5.24 diversity index. The composition of families and trees species are presented in Table 1 and 2.
From Figure 1 and 2, it can be seen that the composition of tree species of Moraceae is higher than the other families which reach 14 and 11 species respectively, followed by Mimosaceae, Poaceae, Myrtaceae, Euphorbiaceae in the first area and Euphorbiaceae, Myrtaceae, Anacardiaceae, Sterculiaceae in the second area. Moraceae is one of the family of flowering plants, the tribe of Rosales. This tribe also includes the genus of Ficus. Most of the Moraceae family grows in the lowland tropics and even the genus of Ficus distribution centers on Indo-Malesia regions including Indonesia, Malaysia, the Philippines, Brunei, and Papua New Guinea. Some Ficus species can be classified as a key species (keystone species) because of its fruit. It is preferred to eat by animals, so it is potential if it is planted as a material for improving the environment quality $[3,4,5,6]$.

In accordance to the restoration and maintenance of water resources, some species of the genus of Ficus have specific characteristics, such as deep and broad rooting, many branches in low posisition, and broad canopy potential to reduce the speed of rainfall grains. Thus the destructive force on the surface layer of soil is low, and the infiltration of water into the ground is better. As the result, water is retained relatively longer in the soil and is released slowly, allowing the continuity of spring and reducing erosion or landslides [7].

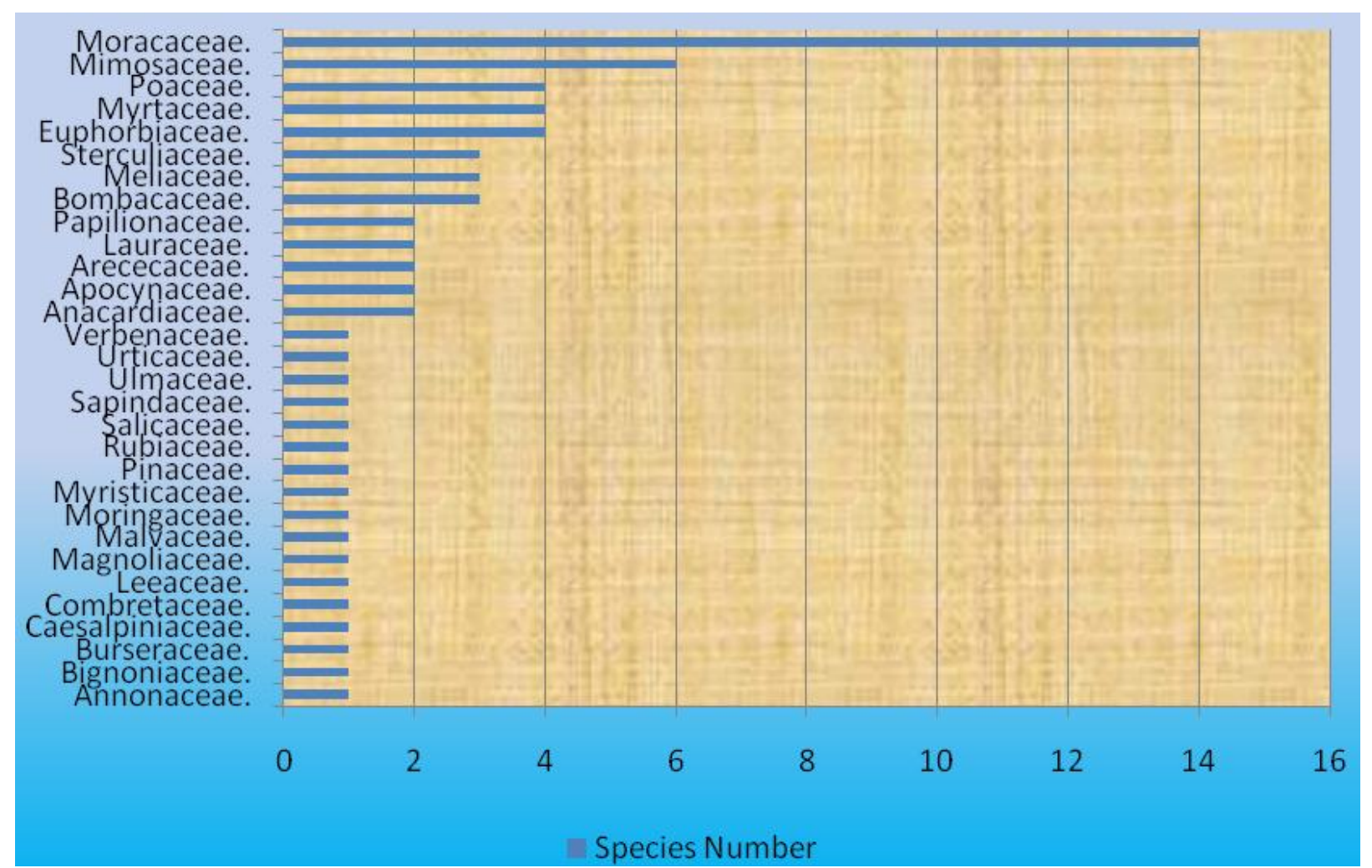

Figure 1. Families composition and Tree species number in the First Area (Sekarmojo-Cendono) 


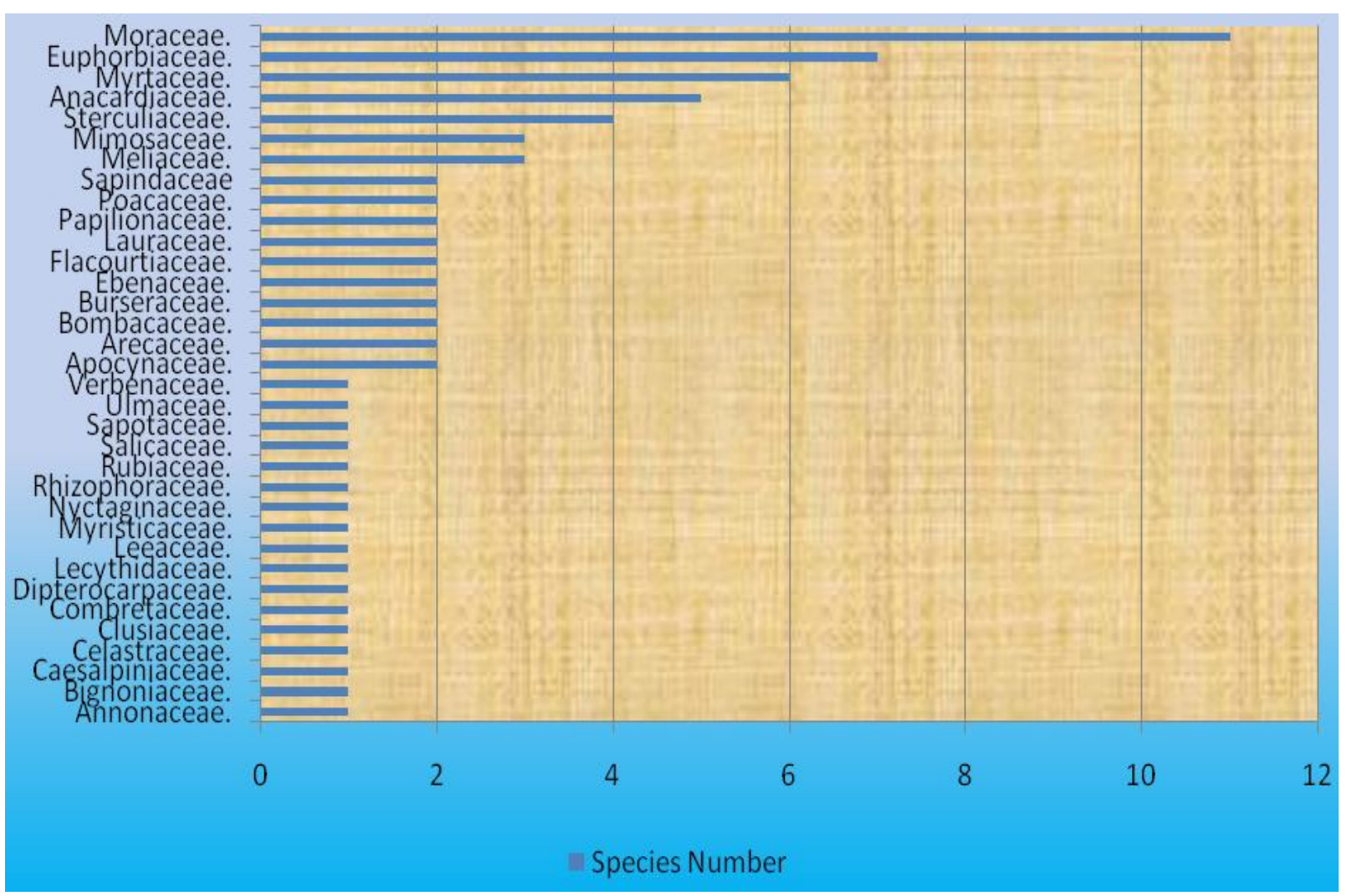

Figure 2. Families composition and Tree species number in the second Area (Pucangsari-Pager-Kucur)

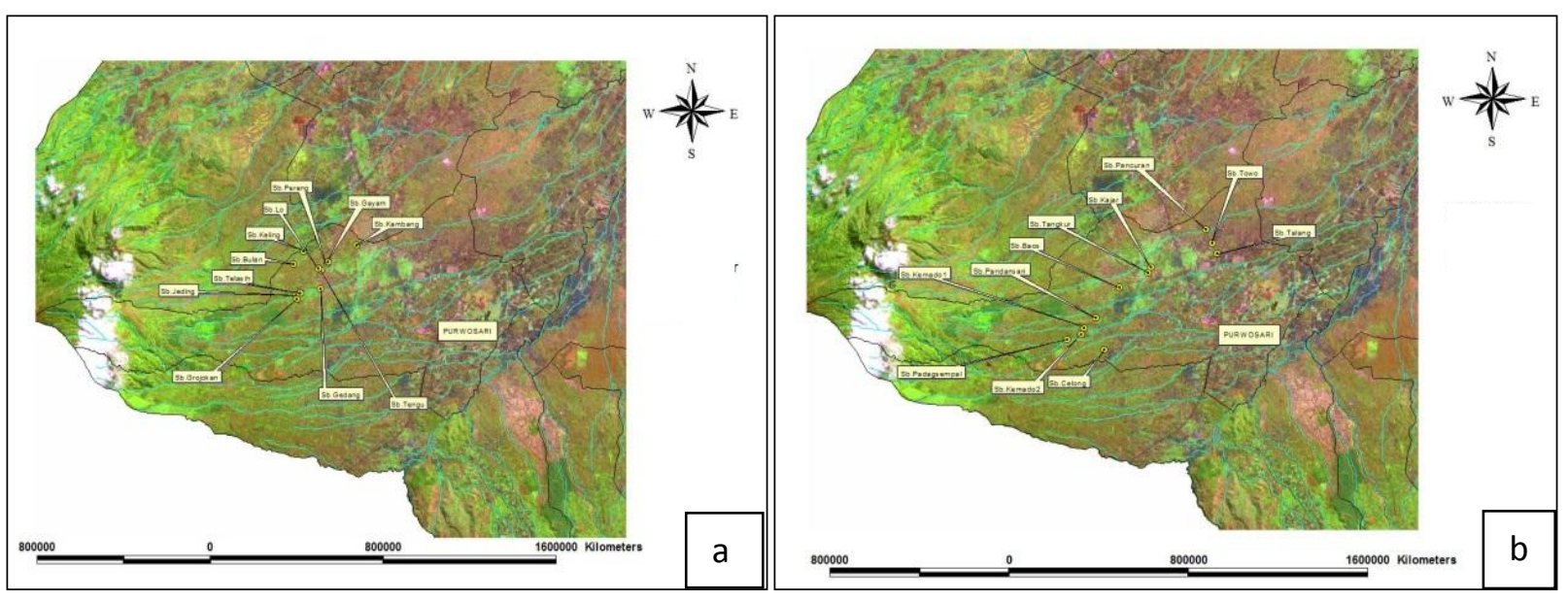

Figure 3. The positions of Springs or Spring groups in Two Areas in the Sub-District of Purwosari (Yellow dot); first area (a); and the second area (b); Map quoted from: Pasuruan SIMTARU.

From Figure 2, there are two families that were quite interesting to inform, namely the Rhizophoraceae and Dipterocarpaceae. Among the better known members of Rhizophoraceae family are mangrove trees, the genus of Rhizophora [8]. Rhizophora species generally live in intertidal zones which are inundated daily by the ocean. They exhibit a number of adaptations to this environment, including stilt-roots that elevate the plants above the water and allow them to respire oxygen even while their lower roots are submerged[9]. Some other genus can be grown on land [8] and the only one species found in this study is Carallia bracbiata (Lour.) Merr. Its habitat generally is in evergreen forest, especially along rivers, below $400 \mathrm{~m}$ [10;11].

In this study, C. brachiata was found on the riverbank at an altitude of $256 \mathrm{~m}$ above sea level. The difference between the mangrove species and other species that typically grow in the land can be seen from the character of the seeds. Mangrove species are usually viviparous while those living on land are not[8]. Another family is Dipterocarpaceae. It has 17 genera and 
approximately 500 species of mainly tropical lowland rainforest trees. Many are large forest emergent species, typically reaching heights of 40-70 m tall, some even over $80 \mathrm{~m}$ and one of them is genus Hopea. Their distribution is pantropical and the greatest diversity of Dipterocarpaceae occurs in Borneo. Some species are now endangered [12]. In Java there are two species Hopea, H. celebica and H. sangal [13]. Both species are now rarely found in Java[13]. In more detail, according to[14] and [15], the population of $H$. celebica is endangered $\mathrm{A} 1 \mathrm{~cd}+2 \mathrm{~cd}, \mathrm{~B} 1+2 \mathrm{c}$ ver 2.3 while $H$. sangal is critically endangered $\mathrm{A} 1 \mathrm{~cd}, \mathrm{~B} 1+2 \mathrm{c}, \mathrm{C} 1, \mathrm{D}$ ver 2.3. H. Sangal generally spreads on clay-rich soil on river banks or hillsides up to $500 \mathrm{~m}[16]$. The only species and the only specimen of the family Dipterocarpaceae found in this study is $H$. sangal. It was found on the edge of the water source at an altitude of $522 \mathrm{~m}$ above sea level. Some seeds have been collected and been successfully germinated in the nursery of the Purwodadi Botanical Gardens.

Other species that have also been collected and been successfully germinated in the nursery are Horsfieldia irya (Myristicaceae) and Baringtonia insignis (Lecythidaceae). The later three species, Hopea sangal, Horsfieldia irya and Baringtonia insignis were collected by the Botanical Gardens. Therefore, those plants are expected to be added as new collection at the Purwodadi Botanical Gardens. I think this work provides sufficient information valueable for the conservation interests, either in national or international standards, especially when viewed from the standpoint of the basic tasks and functions of botanical gardens as well as international conservation targets such as conservation targets that are packed in the Global Strategy for Plant Corservation [17].

Based on Table 1 and 2, it can be seen that the composition of tree species with the highest importance value index, both in the first and the second area, was Bambusa blumeana from Poaceae. This is understandable because bamboo typically grows in clumps; thus affecting the number of individuals in each plot observation. Consequently, this would influence the significance of the important value index. Bambusa blumeana is commonly known growing in tropical Asia[18]. Unless Bambusa blumeana, there are also some species that are co-dominant such as, Dendrocalamus asper, Ficus racemosa, Horsfieldia irya, and Ficus virens. Dendrocalamus asper which also grow in clumps, while the significance of Ficus racemosa, Horsfieldia irya, and Ficus virens is more influenced by their stem diameter, which on average is quite large.

\section{The Spring Position on the Map}

The positions of the springs in the two areas within the sub-districts of Purwosari are in the range of $7^{\circ} 44^{\prime} 448$ " south latitude; $112^{\circ} 44^{\prime} 353^{\prime \prime}$ longitude up to $7^{\circ} 46^{\prime} 339$ " south latitude; $112^{\circ} 41^{\prime} 190^{\prime \prime}$ longitude at an altitude between 251 and $522 \mathrm{~m}$ above sea level. The position of the spring and the spring groups are listed in Figure $3 a b$.

\section{CONCLUSION}

At least 30 families, 49 genera which consisted of 68 species of trees grown at surrounding water springs at the first area with 5.49 of diversity index, while the second area consisted of 34 families, 63 genera and 79 species of trees with 5.24 diversity index. The composition of trees species from Moraceae was the highest among other families, both at the first and the second area, whereas trees species having a significant important value index include Bambusa blumeana, Dendrocalamus asper, Ficus racemosa, Horsfieldia irya and Ficus virens. The positions of the springs in the two areas within the sub-districts of Purwosari are in the range of $7^{\circ} 44^{\prime} 448^{\prime \prime}$ south latitude; 112 44'353" east longitude up to $7^{\circ} 46^{\prime} 339^{\prime \prime}$ south latitude; $112^{\circ} 41^{\prime} 190^{\prime \prime}$ east longitude at an altitude between 251 and $522 \mathrm{~m}$ above sea level. From this study it was recorded that at least three species, Hopea sangal, Horsfieldia irya and Baringtonia insignis are expected to be added as new collections at Purwodadi Botanical Garden.

\section{ACKNOWLEDGEMENTS}

The author would like to thank Mr. Sugiyana and Haryono which have helped the activities in the field and Mr. Adi Laksono A.Md. who helped to analyze the data in the GPS so they can be included in this papers.

\section{REFERENCES}

1. Marinelli J (2004) Plant. The Ultimate Visual Reference to plants and flowers of the world, Royal Botanic Gardens. Kew.

2. Soerianegara I \& A Indrawan (1983) Ekologi Hutan Indonesia. Departemen manajemen 
Hutan, fakultas Kehutanan Institut Pertanian Bogor. Bogor.

3. Backer CA \& RC Bakhuizen van den Brink Jr. (1965) Flora of Java Vol. II. N.V.P. Noordhoff Groningen. Netherlands.

4. Berg CC \& EJH. Corner (2005) Flora Malesiana Series I-Seed Plants Vol 17/ Part 2-2005, Moraceae (Ficus). National Herbarium Nederland.

5. Sastrapradja S \& JJ Afriastini (1984) Kerabat Beringin. Seri Sumber Daya Alam 115. Lembaga Biologi Nasional-LIPI. Bogor.

6. Widyatmoko D \& Irawati (2007) Kamus Istilah Konservasi. Pusat Konservasi Tumbuhan Kebun Raya Bogor. LIPI Press. Jakarta.

7. Soejono (2011) The Trees Species Diversity Around Spring Water At Two Areas In Purwodadi, Pasuruan. Proceeding international Coference on Biological Science Advances in Biological science. Faculty Of biology Universitas Gajah mada Yogyakarta. September 23rd-24th

8. Anonimous (2012) Rhizoporaceae, http://en. wikipedia.org /wiki/Rhizophoraceae. Accessed on June 28th 2012.

9. Anonymous (2012) Rhizophora, http://en. wikipedia.org/wiki/Rhizophora. Accessed on June 282012.

10. Anonymous (2012) Carallia brachiata (Lour.) Merr. Rhizophoraceae. Botanical Descriptions Habitat And Ecology Distribution, http://www.biotik.org/laos/species/c/carbr/car br_en.html. Accessed on June 28th 2012.

11. Backer CA \& RC Bakhuizen van den Brink Jr. (1963) Flora of Java Vol. I. N.V.P. Noordhoff Groningen, Netherlands.

12. Anonymous (2012) Dipterocarpaceae, http://en. wikipedia.org/wiki/Dipterocarpaceae, Accessed on June 28th 2012.

13. Heyne K (1987) Tumbuhan Berguna Indonesia, Jilid III (Terjemahan), Badan Litbang Kehutanan, Jakarta.

14. Ashton P (1998) Hopea celebica, In: IUCN 2012. IUCN Red List of Threatened Species, Version 2012. www.iucnredlist.org. Accessed on July 30th 2012.

15. Soerianegara \& RHMJ Lemmens (1994) Plant Resources of South East Asia 5 (1) Timber Trees: Major Commersial Timbers. Prosea Foundation. Bogor. Indonesia.
16. Zedan H. (2002) Global Strategy for Plant Conservation. Botanic Gardens Conservation International Descanso House. 199 Kew Road Richmond, Surrey TW9 3BW. UK.

17. Anonymous (2011) Bamboo, http://en. wikipedia.org/wiki/Bambusa_blumeana, Accessed on March 22nd 2011.

18. Anonymous (2009) Simtaru Pasuruan, Pemerintah Kabupaten Pasuruan, Pasuruan. Unpublished. 


\section{Supplementary Data}

Table 1. Tree Species composition and Importance Value Index at the First area (Sekarmojo-Cendono)

\begin{tabular}{|c|c|c|c|c|c|c|c|}
\hline No. & Species & Family & $\mathbf{F R}$ & $\mathbf{K R}$ & DR & IV & $\mathbf{H i}$ \\
\hline 1 & Bambusa blumeana Schult.f. & Poac. & 4.88 & 30.22 & 5.27 & 40.370 & 1.220 \\
\hline 2 & Dendrocalamus asper (Roem. \& Schult.f.) Backer ex Heyne & Poac. & 4.07 & 27.75 & 5.77 & 37.582 & 1.142 \\
\hline 3 & Ficus racemosa $\mathrm{L}$. & Morac. & 3.25 & 0.96 & 25.59 & 29.802 & 0.089 \\
\hline 4 & Syqygium javanicum Miq. & Myrt. & 2.44 & 1.79 & 8.24 & 12.463 & 0.121 \\
\hline 5 & Syøygium picnanthum Merr. \& L.M. Perry & Myrt. & 4.88 & 4.26 & 3.12 & 12.261 & 0.288 \\
\hline 6 & Ceiba pentandra (L.) Gaertn. & Bombac. & 4.07 & 3.71 & 4.10 & 11.874 & 0.257 \\
\hline 7 & Alstonia scholaris (L.) R. Br. & Apoc. & 2.44 & 1.10 & 6.32 & 9.859 & 0.083 \\
\hline 8 & Saraca indica $L$. & Caesal. & 1.63 & 3.85 & 3.87 & 9.338 & 0.200 \\
\hline 9 & Dysoxylum gaudichaudianum (A. Juss.) Miq. & Meliac. & 3.25 & 1.24 & 4.53 & 9.022 & 0.107 \\
\hline 10 & Arenga pinnata (Wurmb) Merr. & Arec. & 4.88 & 1.79 & 2.29 & 8.952 & 0.147 \\
\hline 11 & Ficus benjamina L. & Morac. & 1.63 & 0.41 & 6.19 & 8.227 & 0.036 \\
\hline 12 & Artocarpus elasticus Reinw. Ex Blume & Morac.. & 2.44 & 0.69 & 1.71 & 4.834 & 0.060 \\
\hline 13 & Ficus kurziii King & Morac. & 1.63 & 0.69 & 2.34 & 4.650 & 0.056 \\
\hline 14 & Tectona grandis L.f. & Verb. & 2.44 & 1.10 & 0.27 & 3.807 & 0.089 \\
\hline 15 & Pinus merkusii Jungh \& de Vriese & Pinac. & 0.81 & 2.06 & 0.92 & 3.791 & 0.115 \\
\hline 16 & Ficus variegata $B l$. & Morac. & 2.44 & 0.41 & 0.74 & 3.587 & 0.039 \\
\hline 17 & Dendrocnide stimulans (L.f.) Chew & Urt. & 1.63 & 0.55 & 1.18 & 3.352 & 0.046 \\
\hline 18 & Syaygium polyanthum (Wight) Walp & Myrt. & 1.63 & 0.96 & 0.62 & 3.203 & 0.073 \\
\hline 19 & Sapindus rarak DC. & Sapind. & 2.44 & 0.41 & 0.22 & 3.075 & 0.039 \\
\hline 20 & Persea americana Mill. & Laur. & 1.63 & 0.96 & 0.18 & 2.763 & 0.074 \\
\hline 21 & Albizia chinensis (Osb.) Merr. & Fab. & 1.63 & 0.55 & 0.54 & 2.713 & 0.046 \\
\hline 22 & Biscofia javanica Blume & Euph. & 0.81 & 0.41 & 1.46 & 2.690 & 0.033 \\
\hline 23 & Ficus sp. 1 & Morac. & 0.81 & 0.69 & 1.14 & 2.636 & 0.049 \\
\hline 24 & Artocarpus altilis (Park. Ex Zoll.) Forsberg & Morac. & 1.63 & 0.55 & 0.44 & 2.616 & 0.047 \\
\hline 25 & Ficus virens $\mathrm{W}$. Aiton & Morac. & 1.63 & 0.27 & 0.66 & 2.560 & 0.026 \\
\hline 26 & Ficus superba (Miq.) Miq. & Morac. & 0.81 & 0.14 & 1.55 & 2.505 & 0.013 \\
\hline 27 & Swietenia macrophylla King & Meliac. & 0.81 & 1.51 & 0.17 & 2.498 & 0.091 \\
\hline 28 & Gigantochloa atter(Hassk.) kurz ex Munro & Poac. & 0.81 & 1.37 & 0.21 & 2.393 & 0.085 \\
\hline 29 & Terminalia microcarpa Decne & Combret. & 0.81 & 0.14 & 1.43 & 2.383 & 0.013 \\
\hline 30 & Salix tetrastigma Roxb. & Salic. & 1.63 & 0.41 & 0.34 & 2.376 & 0.036 \\
\hline 31 & Spathodea campanulata Beauv. & Bign. & 1.63 & 0.41 & 0.33 & 2.371 & 0.036 \\
\hline 32 & Sterculia coccinea Jack & Sterc. & 0.81 & 0.27 & 1.12 & 2.207 & 0.023 \\
\hline 33 & Sterculia macropbylla Vent. & Sterc. & 0.81 & 0.69 & 0.70 & 2.204 & 0.049 \\
\hline 34 & Bombac ceiba $\mathrm{L}$. & Bombac. & 0.81 & 0.14 & 1.20 & 2.154 & 0.013 \\
\hline 35 & Moringa pterygosperma Gaertn. & Moring. & 1.63 & 0.41 & 0.07 & 2.110 & 0.036 \\
\hline 36 & Cananga odorata (Lam.) Hook.f. \& Thomson & Annon. & 1.63 & 0.27 & 0.18 & 2.085 & 0.026 \\
\hline 37 & Artocarpus beterophyllus Lam. & Morac. & 1.63 & 0.27 & 0.13 & 2.030 & 0.013 \\
\hline 38 & Garuga floribunda Decne & Burs. & 0.81 & 0.27 & 0.83 & 1.916 & 0.023 \\
\hline 39 & Durio aybetbinus Murr. & Bombac. & 0.81 & 0.27 & 0.45 & 1.538 & 0.023 \\
\hline 40 & Glocbidion mole Blume & Euph. & 0.81 & 0.55 & 0.09 & 1.453 & 0.041 \\
\hline 41 & Ficus rumphii Blume & Morac.. & 0.81 & 0.41 & 0.21 & 1.439 & 0.033 \\
\hline 42 & Horsfieldia irya (Gaertn) Warb. & Myrist. & 0.81 & 0.27 & 0.29 & 1.376 & 0.023 \\
\hline
\end{tabular}


Trees Grown Surrounding Water Springs at Purwosari

\begin{tabular}{|c|c|c|c|c|c|c|c|}
\hline 43 & Leucaena leucocephalla (Lam.) de Wit & Mim. & 0.81 & 0.41 & 0.13 & 1.357 & 0.033 \\
\hline 44 & Lansium domesticumCorr. & Meliac. & 0.81 & 0.41 & 0.09 & 1.319 & 0.033 \\
\hline 45 & Ficus retusa Auct. Non L & Morac.. & 0.81 & 0.14 & 0.36 & 1.308 & 0.013 \\
\hline 46 & Lannea coromandelica (Houttt.) Merr. & Anac. & 0.81 & 0.14 & 0.36 & 1.308 & 0.013 \\
\hline 47 & Adenanthera pavonina $L$. & Fab. & 0.81 & 0.14 & 0.25 & 1.199 & 0.013 \\
\hline 48 & Parkia speciosa Hassk. & Mim. & 0.81 & 0.27 & 0.10 & 1.190 & 0.023 \\
\hline 49 & Erythrina subumbrans (Hassk.) Merr. & Papil. & 0.81 & 0.27 & 0.08 & 1.167 & 0.023 \\
\hline 50 & Michelia champaca $\mathrm{L}$. & Magnol. & 0.81 & 0.27 & 0.06 & 1.150 & 0.023 \\
\hline 51 & Leea angulata Korth. Ex Miq. & Leeac. & 0.81 & 0.27 & 0.05 & 1.142 & 0.023 \\
\hline 52 & Parkia timorriana (DC.) Merr. & Mim. & 0.81 & 0.27 & 0.05 & 1.136 & 0.023 \\
\hline 53 & Ficus callosa Willd. & Morac. & 0.81 & 0.14 & 0.16 & 1.110 & 0.013 \\
\hline 54 & Hibiscus tiliaceus L. & Malv. & 0.81 & 0.14 & 0.16 & 1.110 & 0.013 \\
\hline 55 & Pterocarpus indicus Willd. & Papil. & 0.81 & 0.14 & 0.16 & 1.110 & 0.013 \\
\hline 56 & Syzygium cumini (L.) Skeels & Myrt. & 0.81 & 0.14 & 0.16 & 1.110 & 0.013 \\
\hline 57 & Ficus elastica Nois. Ex Bl. & Morac. & 0.81 & 0.14 & 0.12 & 1.072 & 0.013 \\
\hline 58 & Pimelodendron amboinicum Hassk. & Euph. & 0.81 & 0.14 & 0.12 & 1.072 & 0.013 \\
\hline 59 & Mangifera indica $\mathrm{L}$. & Anac. & 0.81 & 0.14 & 0.09 & 1.040 & 0.013 \\
\hline 60 & Melanolepis multiglandulosa Reich.f. & Euph. & 0.81 & 0.14 & 0.09 & 1.040 & 0.013 \\
\hline 61 & Trema orientalis (L.) Bl. & Ulm. & 0.81 & 0.14 & 0.09 & 1.040 & 0.013 \\
\hline 62 & Litsea noronhae Bl. & Laur. & 0.81 & 0.14 & 0.06 & 1.013 & 0.013 \\
\hline 63 & Morinda citrifolia $\mathrm{L}$. & Rub. & 0.81 & 0.14 & 0.06 & 1.013 & 0.013 \\
\hline 64 & Albizia procera (Roxb.) Benth. & Mim. & 0.81 & 0.14 & 0.04 & 0.990 & 0.013 \\
\hline 65 & Areca cathecu L. & Arec. & 0.81 & 0.14 & 0.04 & 0.990 & 0.013 \\
\hline 66 & Sterculia cordata Blume & Sterc. & 0.81 & 0.14 & 0.04 & 0.990 & 0.013 \\
\hline 67 & Tabernaemontana sphaerocaerpa Blume & Apoc. & 0.81 & 0.14 & 0.04 & 0.990 & 0.013 \\
\hline 68 & Baubinia malabarica Roxb. & Poac. & 0.81 & 0.14 & 0.02 & 0.973 & 0.013 \\
\hline
\end{tabular}

Description: KR: Relative Density; DR: Relative dominance; FR: Relative Frequency; IV: Impotance value Index; Hi: Diversity Index 
Table 2. Tree Species composition and Importance Value Index at the Second area (Pucangsari-Pager-Kucur)

\begin{tabular}{|c|c|c|c|c|c|c|c|}
\hline No. & Species & Family & FR & $\mathbf{K R}$ & DR & IV & $\mathbf{H i}$ \\
\hline 1 & Bambusa blumeana Schult.f. & Poac. & 2.14 & 39.67 & 3.01 & 44.819 & 1.029 \\
\hline 2 & Horsfieldia irya (gaertn.) Warb. & Myrist. & 2.86 & 4.86 & 10.91 & 18.635 & 0.278 \\
\hline 3 & Ficus virens $\mathrm{W}$. Aiton & Morac. & 2.14 & 0.91 & 15.24 & 18.298 & 0.075 \\
\hline 4 & Ceiba pentandra (L.) Gaertn. & Bombac. & 1.43 & 3.95 & 7.26 & 12.642 & 0.200 \\
\hline 5 & Barringtonia insignis (Bl.) Miq. & Lecyth. & 1.43 & 5.47 & 1.86 & 8.757 & 0.268 \\
\hline 6 & Ficus benjamina L. & Morac. & 2.14 & 0.46 & 6.02 & 8.618 & 0.043 \\
\hline 7 & Artocarpus elasticus Reinw. Ex Blume & Morac. & 2.86 & 1.82 & 3.58 & 8.258 & 0.138 \\
\hline 8 & Alstonia scholaris (L.) R. Br. & Apoc. & 2.86 & 0.61 & 4.41 & 7.876 & 0.057 \\
\hline 9 & Syzygium syaygioides (Miq.) Amshoff & Myrt. & 0.71 & 0.30 & 5.29 & 6.309 & 0.025 \\
\hline 10 & Diospyros haseltii Zoll. & Eben. & 2.14 & 2.74 & 1.31 & 6.193 & 0.176 \\
\hline 11 & Dracontomelon dao (Blanco) Merril \& Rolfe & Anac. & 1.43 & 1.67 & 2.85 & 5.948 & 0.115 \\
\hline 12 & Glocbidion mole Blume & Euph. & 2.86 & 1.98 & 0.90 & 5.734 & 0.134 \\
\hline 13 & Syzygium picnanthum Merr. \& L.M. Perry & Myrt. & 2.86 & 2.13 & 0.72 & 5.709 & 0.159 \\
\hline 14 & Buchanania arborescens (Blume) Blume & Anac. & 2.86 & 1.37 & 1.39 & 5.613 & 0.111 \\
\hline 15 & Areca cathecu $\mathrm{L}$. & Arec. & 2.14 & 3.19 & 0.26 & 5.590 & 0.202 \\
\hline 16 & Syzygium littorale (Blume) Amshoff & Myrt. & 2.86 & 1.22 & 1.15 & 5.220 & 0.099 \\
\hline 17 & Dendrocalamus asper (Schult.f.) Backer ex Heyne & Poac. & 0.71 & 3.80 & 0.54 & 5.058 & 0.179 \\
\hline 18 & Dysoxylum gaudichaudianum (A.Juss.) Miq. & Meliac. & 2.86 & 0.76 & 1.43 & 5.047 & 0.068 \\
\hline 19 & Sterculia foetida $\mathrm{L}$. & Sterc. & 1.43 & 1.22 & 2.34 & 4.986 & 0.084 \\
\hline 20 & Parkia timoriana (DC.) Meer. & Mim. & 1.43 & 0.46 & 2.79 & 4.674 & 0.040 \\
\hline 21 & Anthocephalus chinensis (Lam.) Rich. Ex Walp & Rub. & 2.14 & 1.06 & 1.39 & 4.592 & 0.082 \\
\hline 22 & Terminalia microcarpa Decne & Combret. & 2.14 & 0.46 & 1.61 & 4.211 & 0.043 \\
\hline 23 & Bischofiaa javanica $\mathrm{Bl}$. & Euph. & 1.43 & 0.91 & 1.81 & 4.154 & 0.071 \\
\hline 24 & Syzygium polyanthum (Wight) Walp & Myrt. & 2.86 & 0.76 & 0.22 & 3.837 & 0.068 \\
\hline 25 & Ficus racemosa $\mathrm{L}$. & Morac. & 0.71 & 0.46 & 2.65 & 3.817 & 0.035 \\
\hline 26 & Ficus variegata Blume & Morac. & 2.14 & 0.61 & 0.62 & 3.370 & 0.054 \\
\hline 29 & Swietenia macrophylla King & Meliac. & 0.71 & 1.82 & 0.77 & 3.311 & 0.105 \\
\hline 30 & Antaris toxicaria (Pers.) Lesch & Morac. & 1.43 & 0.61 & 1.07 & 3.102 & 0.050 \\
\hline 31 & Inocarpus fagifera (Park.) Fosb. & Papil. & 1.43 & 0.46 & 1.04 & 2.922 & 0.040 \\
\hline 32 & Tabernaemontana spaerocarpa Blume & Apoc. & 1.43 & 1.06 & 0.37 & 2.862 & 0.076 \\
\hline 33 & Pangium edule Reinw. & Flac. & 1.43 & 0.46 & 0.85 & 2.737 & 0.040 \\
\hline 34 & Pometia pinnata J.R. \& G. Forst var javanica K. \& V. & Sapind & 1.43 & 0.30 & 0.99 & 2.721 & 0.028 \\
\hline 35 & Garuga floribunda Decne & Burs. & 1.43 & 0.46 & 0.77 & 2.655 & 0.040 \\
\hline 36 & Artocarpus altilis (Park. Ex Zoll.) Forsberg (sukun) & Morac. & 1.43 & 0.76 & 0.42 & 2.608 & 0.061 \\
\hline 37 & Syzygium cumini (L.) Skeels & Myrt. & 1.43 & 0.46 & 0.28 & 2.167 & 0.040 \\
\hline 38 & Antidesma bunius (L.) Spreng. & Euph. & 1.43 & 0.46 & 0.25 & 2.132 & 0.040 \\
\hline 39 & Arenga pinnata (Wurmb) Meer. & Arec. & 0.71 & 0.76 & 0.64 & 2.110 & 0.054 \\
\hline 40 & Sterculia macrophylla Vent. & Sterc. & 0.71 & 0.61 & 0.76 & 2.081 & 0.045 \\
\hline 41 & Palaquium amboinense Burck & Sapot. & 1.43 & 0.46 & 0.19 & 2.072 & 0.040 \\
\hline 42 & Cananga odorata (lam.) Hook.f.\& Thomson & Annon. & 1.43 & 0.46 & 0.15 & 2.036 & 0.040 \\
\hline 43 & Ficus sp. & Morac. & 0.71 & 0.15 & 1.15 & 2.018 & 0.014 \\
\hline 45 & Persea americana Mill. & Laur. & 1.43 & 0.46 & 0.07 & 1.954 & 0.040 \\
\hline 46 & Aphanamixis grandifolia (Blume) Walp. & Meliac. & 1.43 & 0.30 & 0.20 & 1.930 & 0.028 \\
\hline
\end{tabular}


Trees Grown Surrounding Water Springs at Purwosari

\begin{tabular}{|c|c|c|c|c|c|c|c|}
\hline 47 & Flacourtia rukam Zoll. \& Moritzi & Flac. & 1.43 & 0.30 & 0.08 & 1.809 & 0.028 \\
\hline 48 & Artocarpus heterophyllus Lam. & Morac. & 1.43 & 0.30 & 0.07 & 1.802 & 0.028 \\
\hline 49 & Siphonodon celastrineus Griff. & Celast. & 0.71 & 0.61 & 0.46 & 1.782 & 0.045 \\
\hline 50 & Hopea sangal Korth. & Dipt. & 0.71 & 0.15 & 0.82 & 1.691 & 0.014 \\
\hline 51 & Spathodea campanulata Beauv. & Bign. & 0.71 & 0.15 & 0.82 & 1.691 & 0.014 \\
\hline 52 & Sterculia coccinea Jack & Sterc. & 0.71 & 0.15 & 0.82 & 1.691 & 0.014 \\
\hline 53 & Pisonia exelsa $\mathrm{Bl}$. & Nyct. & 0.71 & 0.61 & 0.32 & 1.637 & 0.045 \\
\hline 54 & Gluta renghas $\mathrm{L}$. & Anac. & 0.71 & 0.46 & 0.31 & 1.476 & 0.035 \\
\hline 55 & Erythrina fusca Lour. & Papil. & 0.71 & 0.30 & 0.42 & 1.434 & 0.025 \\
\hline 56 & Aleurites moluccana (L.) Willd. & Euph. & 0.71 & 0.15 & 0.44 & 1.303 & 0.014 \\
\hline 57 & Ficus kurz̨ii King & Morac. & 0.71 & 0.15 & 0.44 & 1.303 & 0.014 \\
\hline 58 & Carallia brachiata (Lour.) Merr. & Rhizoph. & 0.71 & 0.15 & 0.38 & 1.250 & 0.014 \\
\hline 59 & Sterculia cordata Blume & Sterc. & 0.71 & 0.15 & 0.38 & 1.250 & 0.014 \\
\hline 60 & Beilschmiedia roxburghiana Nees & Laur. & 0.71 & 0.30 & 0.19 & 1.211 & 0.025 \\
\hline 61 & Cassia fistula L. & Caesal. & 0.71 & 0.15 & 0.33 & 1.200 & 0.014 \\
\hline 62 & Ficus callophylla $\mathrm{Bl}$. & Morac. & 0.71 & 0.15 & 0.33 & 1.200 & 0.014 \\
\hline 63 & Protium javanicum burm.f. & Burs. & 0.71 & 0.15 & 0.33 & 1.200 & 0.014 \\
\hline 64 & Baccaurea dulcis (Jack) Muell. Arg. & Euph. & 0.71 & 0.30 & 0.17 & 1.189 & 0.025 \\
\hline 65 & Achmena acuminatissima(Blume) Meer. \& L.M. Perry & Myrt. & 0.71 & 0.15 & 0.25 & 1.112 & 0.014 \\
\hline 66 & Albizia procera (Roxb.) Benth. & Mim. & 0.71 & 0.15 & 0.25 & 1.112 & 0.014 \\
\hline 67 & Lannea coromandelica (Houttt.) Merr. & Anac. & 0.71 & 0.30 & 0.04 & 1.061 & 0.025 \\
\hline 68 & Mangifera indica $\mathrm{L}$. & Anac. & 0.71 & 0.15 & 0.17 & 1.037 & 0.014 \\
\hline 69 & Schleichera oleosa (Lour.) Oken & Sapind. & 0.71 & 0.15 & 0.11 & 0.975 & 0.014 \\
\hline 70 & Trema orientalis (L.) Bl. & Ulm. & 0.71 & 0.15 & 0.11 & 0.975 & 0.014 \\
\hline 71 & Durio zybethinus Murr. & Bombac. & 0.71 & 0.15 & 0.08 & 0.950 & 0.014 \\
\hline 72 & Salix tetrastigma Roxb. & Salic. & 0.71 & 0.15 & 0.08 & 0.950 & 0.014 \\
\hline 73 & Diopyros macrophylla Blume & Eben. & 0.71 & 0.15 & 0.06 & 0.928 & 0.014 \\
\hline 74 & Vitex pinnata L. $=V \cdot$ pubescens Vahl. & Verb. & 0.71 & 0.15 & 0.06 & 0.928 & 0.014 \\
\hline 75 & Macaranga tanarius Mull. Arg. & Euph. & 0.71 & 0.15 & 0.04 & 0.909 & 0.014 \\
\hline 76 & Garcinia dulcis (Roxb.) Kurz & Clus. & 0.71 & 0.15 & 0.03 & 0.894 & 0.014 \\
\hline 77 & Leucaena leucocephalla (Lam.) de Wit & Mim. & 0.71 & 0.15 & 0.03 & 0.894 & 0.014 \\
\hline 78 & Melanolepis multiglandulosa Reich.f. & Euph. & 0.71 & 0.15 & 0.03 & 0.894 & 0.014 \\
\hline 79 & Leea aculeata Blume ex spreng. & Leeac. & 0.71 & 0.15 & 0.02 & 0.882 & 0.014 \\
\hline
\end{tabular}

Description: KR: Relative Density; DR: Relative dominance; FR: Relative Frequency; IV: Impotance value Index; Hi: Diversity Index 